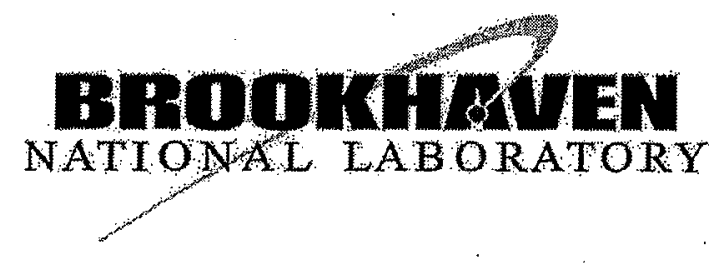

BNL-75398-2006-CP

\title{
Single Spin Asymmetry and Quark Orbital Motion in Nucleon
}

Feng Yuan

Presented at PANIC 05, Particles And Nuclei International Conference, Santa Fe, New Mexico To appear in AIP Conference Proceedings

October 23-28, 2005

January 2006

\author{
Physics Department/ RIKEN BNL Research Center \\ Brookhaven National Laboratory \\ P.O. Box 5000 \\ Upton, NY 11973-5000 \\ www.bnl:gov
}

Notice: This manuscript has been authored by employees of Brookhaven Science Associates, LLC under Contract No. DE-AC02-98CH10886 with the U.S. Department of Energy. The publisher by accepting the manuscript for publication acknowledges that the United States Government retains a non-exclusive, paid-up, irrevocable, world-wide license to publish or reproduce the published form of this manuscript, or allow others to do so, for United States Government purposes.

This preprint is intended for publication in a journal or proceedings. Since changes may be made before publication, it may not be cited or reproduced without the author's permission. 


\section{DISCLAIMER}

This report was prepared as an account of work sponsored by an agency of the United States Government. Neither the United States Government nor any agency thereof, nor any of their employees, nor any of their contractors, subcontractors, or their employees, makes any warranty, express or implied, or assumes any legal liability or responsibility for the accuracy, completeness, or any third party's use or the results of such use of any information, apparatus, product, or process disclosed, or represents that its use would not infringe privately owned rights. Reference herein to any specific commercial product, process, or service by trade name, trademark, manufacturer, or otherwise, does not necessarily constitute or imply its endorsement, recommendation, or favoring by the United States -Government or any agency thereof or its contracters or subcontractors. The views and opinions of authors expressed herein do not necessarily state or reflect those of the United States Government or any agency thereof. 


\title{
Single Spin Asymmetry and Quark Orbital Motion in Nucleon
}

\author{
Feng Yuan \\ RIKEN/BNL Research Center, Building 510A, Brookhaven National Laboratory, Upton, NY 11973
}

\begin{abstract}
In this.talk, we will discuss the single transverse spin asymmetries and their relations to the quark-orbital-angular-momentum of the nucleon.
\end{abstract}

As an important phenomenon, the single (transverse) spin asymmetries (SSAs) in high energy hadronic reactions, which have been discovered for more than two decades, attracted much attention recently from both the theoretical and experimental sides. If the underlying mechanism is hard parton-parton scattering, the SSA was found to be very small [1]. This is because, the hadron helicity flip required by the SSA is suppressed by the current quark mass $\left(m_{q}\right)$, in addition to the suppression due to the phase which can only be generated from hard loop in the partonic process and is suppressed by $\alpha_{s}$. So, in general, the SSAs in these hard parton-parton scattering is in order of $\alpha_{s} m_{q} / Q$ [1] where $Q$ is the typical hard scale in the process (e.g., the invariant mass of the virtual photon for DIS and Drell-Yan processes).

In order to understand these large SSAs observed by various experiments, there have been proposed two mechanisms beyond the naive parton model but still in the QCD framework: one is the twist-3 contribution from the quark-gluon-quark correlations in nucleon $[2,3]$; the other is the transverse momentum dependent (TMD) parton distributions. In this talk, I will focus on the TMD approach for the SSAs, and their relations to the quark orbital angular momentum in nucleon. Parton's intrinsic transverse momentum is crucial to generate large SSAs, because we want to have nonzero quarkorbital-angular-momentum to flip the hadron helicity for single transversely polarized scattering. Once the nonzero quark-orbital-angular momentum is playing role in the scattering, the incident quark will move not only in the longitudinal direction, but also in the transverse direction, which means their transverse momenta have to be taken into account in the scattering. Over the last few years, the gauge properties of the TMD parton distributions have been investigated $[4,5,6,7]$. More recently, the factorization theorems for the semi-inclusive deep inelastic scattering (SIDIS) and Drell-Yan processes have also been re-examined in the context of the gauge-invariant definitions $[4,8,9]$.

The TMD parton distributions can be defined as matrix elements between proton states, with various projection operators depending on their spin and momenta. In the leading twist expansion, there are eight independent TMD parton distributions, and they all contribute to the semi-inclusive DIS and Drell-Yan processes at low transverse momentum in the leading order of $1 / Q$. The light-cone gauge link and the transverse gauge link play very important roles for the gauge invariant definition of these TMD 
parton distributions, which lead to nonzero single spin asymmetries arising from these TMDs.

The Sivers function [10] is one of these interesting TMD parton distributions. It represents a distribution of unpolarized quark in a transversely polarized nucleon, through a correlation between the quark's transverse momentum $\vec{k}_{\perp}$ and the nucleon polarization vector $\vec{S}_{\perp}$. The existence of the Sivers function requires final/initial-state interactions, and an interference between different helicity Fock states of the nucleon. Interference between different helicity Fock states implies nonzero orbital angular momentum $[5,11]$. For example, by explicit calculation, it has been shown that the Sivers function can be expressed in terms of the light-cone wave function overlap between the zero-quarkorbital-angular-momentum and nonzero-quark-orbital-angular-momentum components [11],

$$
q_{T}\left(x, k_{\perp}\right) \propto \frac{M}{k_{\perp}^{2}} \int \operatorname{Im}\left[\tilde{\psi}_{j}^{(1,2) *} \tilde{\psi}_{j}^{(3,4)}+\cdots\right],
$$

where $\tilde{\psi}^{(1,2)}$ are the three-quark light-cone wave functions for the Fock state component of the proton with zero quark-orbital angular momentum projection, and $\tilde{\psi}^{(3,4)}$ are those for the one unit quarrk-orbital-angular-momentum components. The model calculations also demonstrated that the quark-orbital-angular-momentum plays crucial role for nonvanish of the Sivers function [5, 12].

Experimentally, the single spin asymmetries, in particular, in the semi-inclusive deep inelastic scattering have been under intense investigation during last few years. Substantial asymmetries have been reported. Since then, the theoretical attempts have been made to understand these asymmetries and try to extract the Sivers functions, e.g., see a recent summary of these extractions [13]. These studies were based on the Factorization theorems $[4,8,9]$ proven to leading power in the photon virtuality $Q$. The extracted Sivers functions are then used to predict the SSA in the Drell-Yan (DY) process, hopefully to be explored experimentally at RHIC, COMPASS and the GSI. Comparisons of SIDIS and the DY process will be particularly important for testing our understanding of the underlying physics, since it has been predicted $[6,7]$ that the Sivers functions appear with opposite signs in these two processes.

As an example of these fits, in [14], it was assumed that the final hadron's transverse momentum is entirely due to the transverse-momentum dependence in the Sivers function. There is then no further assumption on the particular form of this dependence; rather it is integrated out in order to compare to the experimental data. The transverse momenta contributed by the other factors in the factorization formula will give some smearing effects which may be viewed as "sub-dominant". (However, we emphasize that this will not be true toward small $z$ where the transverse momentum in the fragmentation functions will become important, likely resulting in a suppression of the asymmetry at small z.) The "1/2-moments" of the Sivers functions were then introduced in [14] in the fit to the experimental data. In the actual fit, we have assumed the following simple models for the Sivers function,

$$
\begin{array}{rll}
\text { Model I : } & u_{T}^{(1 / 2)}(x) / u(x)=S_{u} x(1-x), & d_{T}^{(1 / 2)}(x) / u(x)=S_{d} x(1-x), \\
\text { Model II }: & u_{T}^{(1 / 2)}(x) / u(x)=S_{u} x(1-x), & d_{T}^{(1 / 2)}(x) / d(x)=S_{d} x(1-x) .
\end{array}
$$


The difference of these two models is that in model I we parameterize both up and down quark Sivers function in terms of the unpolarized u-quark distribution, while in model II the d-quark Sivers function is parameterized in terms of unpolarized d-quark distribution. By fitting to the HERMES most recent data, we got the following values for the parameters,

$$
\begin{aligned}
\text { Model I : } & S_{u}=-0.81 \pm 0.07, \quad S_{d}=1.86 \pm 0.28, \\
\text { Model II : } & S_{u}=-0.75 \pm 0.07, \quad S_{d}=2.76 \pm 0.46,
\end{aligned}
$$

with similar fit quality: $\chi^{2} / d$.o. $f \sim 1.0$ for both fits. The COMPASS data were not included in the fit performed in[14], but a comparison of the fit with the data was given, showing good agreement. The results of the fit to the HERMES data were furthermore used for making predictions for the SSAs in the Drell-Yan process and in di-jet and jet-photon correlations at RHIC.

Pin down the non-zero quark-orbital-angular-momentum component of the proton wave function is very important. Besides the single spin asymmetry phenomenon discussed in this talk contributing to the understanding of the quark-orbital-angularmomentum of the nucleon, there are also other observables which can be used, such as the nucleon's Pauli form factor $F_{2}$, the spin-dependent structure function $g_{2}$, and the generalized parton distribution $E(x, \xi, t)$. It can help us to determine how much of the nucleon spin is carried by orbital angular momentum. It also help to determine to what extent, the proton is not spherically symmetric (or deformed).

The author is grateful to RIKEN, Brookhaven National Laboratory and the U.S. Department of Energy (contract number DE-AC02-98CH10886) for providing the facilities essential for the completion of his work.

\section{REFERENCES}

1. G. L. Kane, J. Pumplin and W. Repko, Phys. Rev. Lett. 41, 1689 (1978).

2. A. V. Efremov and O. V. Teryaev, Phys. Lett. B 150, 383 (1985).

3. J. Qiu and G. Sterman, Phys. Rev. Lett. 67, 2264 (1991); Phys. Rev. D 59, 014004 (1999).

4. J. C. Collins and D. E. Soper, Nucl. Phys. B 193, 381 (1981) [Erratum-ibid. B 213, 545 (1983)].

5. S. J. Brodsky, D. S. Hwang and I. Schmidt, Phys. Lett. B 530, 99 (2002).

6. J. C. Collins, Phys. Lett. B 536, 43 (2002);

7. X. Ji and F. Yuan, Phys. Lett. B 543, 66 (2002); A. V. Belitsky, X. Ji and F. Yuan, Nucl. Phys. B 656, 165 (2003).

8. X. Ji, J. P. Ma and F. Yuan, Phys. Rev. D 71, 034005 (2005); Phys. Lett. B 597, 299 (2004); JHEP 0507, 020 (2005).

9. J. C. Collins and A. Metz, Phys. Rev. Lett. 93, 252001 (2004).

10. D. W. Sivers, Phys. Rev. D 41, 83 (1990).

11. X. Ji, J. P. Ma and F. Yuan, Nucl. Phys. B 652, 383 (2003).

12. F. Yuan, Phys. Lett. B 575, 45 (2003).

13. M. Anselmino et al., arXiv:hep-ph/0511017.

14. W. Vogelsang and F. Yuan, Phys. Rev. D 72, 054028 (2005). 